The Astrophysical JouRnAL, 486:687-692, 1997 September 10

(C) 1997. The American Astronomical Society. All rights reserved. Printed in U.S.A.

\title{
FEMTOLENS IMAGING OF A QUASAR CENTRAL ENGINE USING A DWARF STAR TELESCOPE
}

\author{
ANDREW GOULD ${ }^{1}$ AND B. SCOTT GAUDI \\ Department of Astronomy, Ohio State University, Columbus, OH 43210; \\ gould@payne.mps.ohio-state.edu, gaudi@payne.mps.ohio-state.edu \\ Received 1996 June 5 ; accepted 1997 April 10
}

\begin{abstract}
We show that it is possible to image the structure of a distant quasar on scales of $\lesssim 1$ AU by constructing a telescope that uses a nearby dwarf star as its "primary lens" together with a satellite-borne "secondary." The image produced by the primary is magnified by $\sim 10^{6}$ in one direction but is contracted by 0.5 in the other and therefore contains highly degenerate one-dimensional information about the two-dimensional source. We discuss various methods for extracting information about the second dimension including "femtolens interferometry" where one measures the interference between different parts of the one-dimensional image with each other. Assuming that the satellite could be dispatched to a position along a star-quasar line of sight at a distance $r$ from the Sun, the nearest available dwarf-star primary is likely to be at $\sim 30 \mathrm{pc}(r / 45 \mathrm{AU})^{-2}$. The secondary should consist of a one-dimensional array of mirrors extending $\sim 170 \mathrm{~m}$ to achieve 1 AU resolution or $\sim 350 \mathrm{~m}$ to achieve $\ll 1$ AU resolution.
\end{abstract}

Subject headings: gravitational lensing — instrumentation: interferometers - quasars: general

\section{INTRODUCTION}

Gravitational microlensing by Galactic stars (and possibly other objects) has been detected in more than 100 events seen toward the Galactic bulge by MACHO (Alcock et al. 1997), OGLE (Udalski et al. 1994), and DUO (Alard 1996). Generally, these stellar lenses are believed to be several kiloparsecs from the Sun, either in the Galactic bulge or in distant regions of the Galactic disk.

There have been several suggestions to make use of more nearby stars as lenses. Nearby stars have high proper motions and relatively large Einstein rings. One could predict when they would lens more distant stars, measure the Einstein ring, and so determine the mass of the lensing star (Refsdal 1964; Paczyński 1995). Even if the projected separation between the nearby and distant stars is many Einstein radii, the former will still deflect the light of the latter. This effect could be measured using interferometry and would again yield a mass measurement (MiraldaEscudé 1996). Extremely nearby objects could conceivably be detected by diffractive lensing flashes (Labeyrie 1994).

Here we propose a more ambitious use of a nearby star as the primary lens of a telescope whose purpose would be to resolve the central engine of a quasar. Since quasars lie at an angular diameter distance $D_{Q} \sim 10^{9} \mathrm{pc}$, and since their central engines are believed to have dimensions $\mathcal{O}(\mathrm{AU})$, such a telescope would require an effective resolution of $10^{-9}$ arcsec, roughly the equivalent of an optical inteferometer with an Earth-Moon baseline. In general, previous suggestions for the use of nearby stars have assumed that one must wait for the lens and source to line up with Earth. The probability for the required alignment with a quasar is negligibly small. However, if Mohammed will not go to the mountain....

\section{CHARACTERISTICS OF THE PRIMARY LENS}

To "construct" the telescope, there must be a dwarf star aligned with a quasar as seen from somewhere in the solar system. This star acts as the "primary lens" of the telescope

\footnotetext{
${ }^{1}$ Alfred P. Sloan Foundation Fellow.
}

while a satellite (described in $\S 3$ ) carries the "secondary optics" required to image the quasar. The dwarf star must have a binary companion with a projected separation $a$ in the range $1 \lesssim a \lesssim 100 \mathrm{AU}$. As we discuss below, this companion induces a slight "astigmatism" in the primary lens that is crucial for the operation of the telescope. For definiteness, we define "somewhere" as being within a distance $r=45 \mathrm{AU}$ of the Sun. For a star at distance $D$, this requirement means that the quasar lies within an angle $r / D$ of the star (as seen from the Sun). The probability of such an occurrence is $\pi(r / D)^{2} N_{Q}$, where $N_{Q}=200 \mathrm{deg}^{-2}$ is the angular density of quasars brighter than $B=22$ (Hartwick $\&$ Schade 1990). The expected number of such alignments by dwarfs within a distance $D_{\text {lim }}$ of the Sun is then

$$
\int_{0}^{D_{\lim }} d D 4 \pi D^{2} \pi\left(\frac{r}{D}\right)^{2} n f_{b} N_{Q}=4 \pi^{2} r^{2} D_{\lim } n f_{\mathrm{b}} N_{Q} \sim \frac{D_{\lim }}{30 \mathrm{pc}},
$$

where we have assumed a local density of dwarfs of $n=0.07$ $\mathrm{pc}^{-3}$ (Gould, Bahcall, \& Flynn 1996 and references therein), and a binary fraction characteristic of $\mathrm{M}$ stars, $f_{b} \sim 0.4$ (Fischer \& Marcy 1992). Even if no such dwarfs are present at a given time, a statistically independent sample will be available after a duration of $r / v \sim 5 \mathrm{yr}$, where we have adopted $v \sim 40 \mathrm{~km} \mathrm{~s}^{-1}$ as the typical transverse speed of disk stars,

We now assume that such a star has been identified at a distance $D \sim 30 \mathrm{pc}$ and has a mass $M \sim 0.4 M_{\odot}$. Since the overwhelming majority of stars have $M_{V}<16$ (Gould et al. 1996), they will appear at $V \lesssim 18$ at $D \lesssim 30 \mathrm{pc}$ and so can be easily identified. We further assume that a satellite has been equipped with the secondary optics that are required to image the quasar (see $\S 3$ ) and that it has been dispatched to a position along the dwarf-quasar line of sight. Note that the satellite must not only be in the correct location but must also be moving with the same transverse velocity as the dwarf star $\left(\sim 40 \mathrm{~km} \mathrm{~s}^{-1}\right)$ so that the alignment with the quasar is maintained.

If the dwarf star were isolated, its lensing properties would be completely characterized by the angular size of its 
Einstein ring $\theta_{e}=\left(4 G M / c^{2} D\right)^{1 / 2}$. For the assumed values of $M$ and $D, \theta_{e} \sim 0.01$, corresponding to $D \theta_{e} \sim 0.3 \mathrm{AU}$. The binary companion will induce a shear

$$
\gamma \sim \frac{m}{M}\left(\frac{D \theta_{e}}{a}\right)^{2} \sim 10^{-4}\left(\frac{20 \mathrm{AU}}{a}\right)^{2},
$$

where $m$ is the mass of the secondary; we have taken $m /$ $M \sim 0.5$. We henceforth assume that $\gamma \sim 10^{-4}$ but discuss other possibilities below. The next largest source of shear is inhomogeneity in the cosmological density of matter. This inhomogeneity induces a shear $\gamma \sim \mathcal{O}(1 \%)$ on images of distant galaxies (Villumsen 1996), but when projected onto the Einstein ring of the dwarf star, the shear is reduced by $\gamma \rightarrow\left(D / D_{c}\right) \gamma \sim 10^{-9}$, where $D_{c} \sim 10^{9} \mathrm{pc}$ is a typical cosmological distance. Other sources of shear are even smaller. For example, the nearest star along the line of sight to the dwarf will lie at an impact parameter $b \sim(n D)^{-1 / 2} \sim 1 \mathrm{pc}$ and will therefore induce a shear $\gamma \sim\left(D \theta_{e} / b\right)^{2} \sim 10^{-11}$.

The shear is approximately constant over the Einstein ring; hence, the dwarf star plus shear is well approximated by a Chang-Refsdal lens (Chang \& Refsdal 1979; Gould \& Loeb 1992; Schneider, Ehlers, \& Falco 1992). Such lenses are completely described by the angular size of the Einstein ring, $\theta_{e}$, the magnitude of the shear, $\gamma$, and the orientation of the shear. The crucial feature of the lens is a diamondshaped caustic (the locus or points with formally infinite magnification), which is centered on the dwarf star and has a diagonal of angular size $\sim 4 \gamma \theta_{e}$. The orientation is given by the position of the secondary. If the binary period is sufficiently short, the mass of both the primary and secondary will be known (since the distance is measured directly from parallax). These in turn determine $\theta_{e}$ and $\gamma$. If the period is long, the satellite can measure $\theta_{e}$ directly by imaging the Einstein ring of the quasar image. The satellite can also determine $\gamma$ by moving between two cusps (the corners of the diamond-shaped caustic) and thereby measuring their projected separation $\left(\sim 4 \gamma \theta_{e} D \sim 2 \times 10^{4} \mathrm{~km}\right)$.

\section{CHARACTERISTICS OF THE SATELLITE}

To resolve the central engine of the quasar, the satellite must situate itself so that the center of the quasar lies inside the caustic and very near the cusp. Let $(\xi, \eta)$ be the coordinates of the source plane in units of the Einstein ring. One may equally well think of these as coordinates of the observer plane with corresponding physical distances $\left(D \theta_{e} \xi\right.$, $\left.D \theta_{e} \eta\right)$. Let $\Delta \xi$ be the distance along the $\xi$ axis from the cusp at $\xi \sim 2 \gamma$. One finds that the caustic in the neighborhood of the cusp is reasonably well described by $\eta^{2}=(\Delta \xi)^{3} / 4 \gamma$. Thus, a source with angular radius $\theta_{*}$ (parameterized by $\left.\rho \equiv \theta_{*} / \theta_{e}\right)$ can come as close as $\Delta \xi \sim\left(4 \gamma \rho^{2}\right)^{1 / 3}$ while still remaining inside the caustic. For sources lying on the $\xi$-axis, the magnification tensor of the brightest image is diagonal, with diagonal components $\left(\Delta \xi^{-1}, 0.5\right)$. Hence, a source of size $\rho \theta_{e} \sim A U / D_{Q} \sim 10^{-9}$ arcsec is stretched by a factor of $\sim 6 \times 10^{5}$ and would therefore subtend an angle $\theta_{r} \sim 0.6$ mas, or $\sim 3.5$ on the Einstein ring.

If the quasar were observed in optical light $(\lambda \sim 0.5 \mu \mathrm{m})$, an effective aperture of $\lambda / \theta_{r} \sim 170 \mathrm{~m}$ would be required to resolve this angle. Below, we will consider what can be accomplished with an aperture of this size, and also with an aperture twice as large, $\sim 350 \mathrm{~m}$. These apertures are smaller than the full width of the caustic, $2 D \rho \theta_{e} \sim 10 \mathrm{~km}$, which implies that the entire effective aperture would fit well inside the caustic projected onto the observer plane. This is an essential ingredient for making the observations. It is impractical and unnecessary to fill the entire aperture with mirrors. In fact, only one dimension must be well sampled because the quasar image is essentially onedimensional: it is highly stretched in one direction and slightly contracted in the other. Even the image of the region $\sim 2000 \mathrm{AU}$ around the quasar would have a lateral extent of only $\sim 1 \mu$ as, far too small to be resolved. Of course, it will be necessary to remove the light from the lensing star, which will be separated from the quasar image by $\theta_{e} \sim 10$ mas and will typically subtend $\sim 0.1$ mas. Most of this light could be directed to a position well off the image center simply by tilting the axis of the array $\sim 30^{\circ}$ relative to the critical curve. There would still be some contamination from the high-order fringes, but much of this could be removed provided that the width of the mirror array was a few tens of meters.

To remain in the caustic over time, the satellite must counter the acceleration due to the Sun at a distance of 45 AU, which is $\sim 4 \times 10^{-4} \mathrm{~cm} \mathrm{~s}^{-1}$. It will also have to counter the acceleration of the binary, which is of the same order. After $10 \mathrm{hr}$ of such acceleration, the satellite will have drifted $\sim 3 \mathrm{~km}$ and will require a boost of $\sim 20 \mathrm{~cm} \mathrm{~s}^{-1}$ to get back on track.

\section{INFORMATION IN ONE AND TWO DIMENSIONS}

The source regions inside the caustic are mapped into four images. For a point source near the cusp, three of these images are highly magnified and lie close to the critical curve on the same side of the lensing star as the quasar. The fourth image lies on the opposite side of the star. It is not highly magnified and will not be considered further. For $\gamma \ll 1$, the critical curve deviates only slightly from the Einstein ring of an isolated lens. The central image is the brightest and lies just outside the critical curve. The other two images lie just inside the critical curve, and the sum of their magnifications is nearly equal to that of the central image. Each image with magnification $A$ is stretched by a factor $2 A$ along the critical curve and compressed by a factor of $0.5 \mathrm{~A}$ perpendicular to it (see Fig. 1).

In principle, since each point of the source is mapped into three unique points of the image, one can reconstruct the source from the image. In fact, all three images are compressed into a one-dimensional curve whose angular width is approximately equal to that of the unlensed source. As mentioned above, this means that a region of $2000 \mathrm{AU}$ is compressed into a curve of width $\sim 1 \mu$ as, far smaller than the resolution element. Thus, in practice each resolution element ("point") along the critical curve represents the sum of the contributions from an entire curve within the source. These "degeneracy curves" are actually almost perfectly straight lines and are tangent to the caustic at exactly one point. Since the resolution element has finite width, the degeneracy curves are in fact "degeneracy bands," whose width shrinks as it approaches the tangent point and then expands again (see Fig. 2).

The nature of this degeneracy can be understood by considering a quasar with a central engine of radius $\sim 1 \mathrm{AU}$, but which is emitting significant amounts of light over a much larger radius $(\sim 200 \mathrm{AU})$. As discussed above, the central AU is imaged into a rectangular arc about 1 mas $\times 10^{-6}$ mas. Other regions of the quasar of intrinsic width $1 \mathrm{AU}$ will be mapped into the same 1 mas, also with 


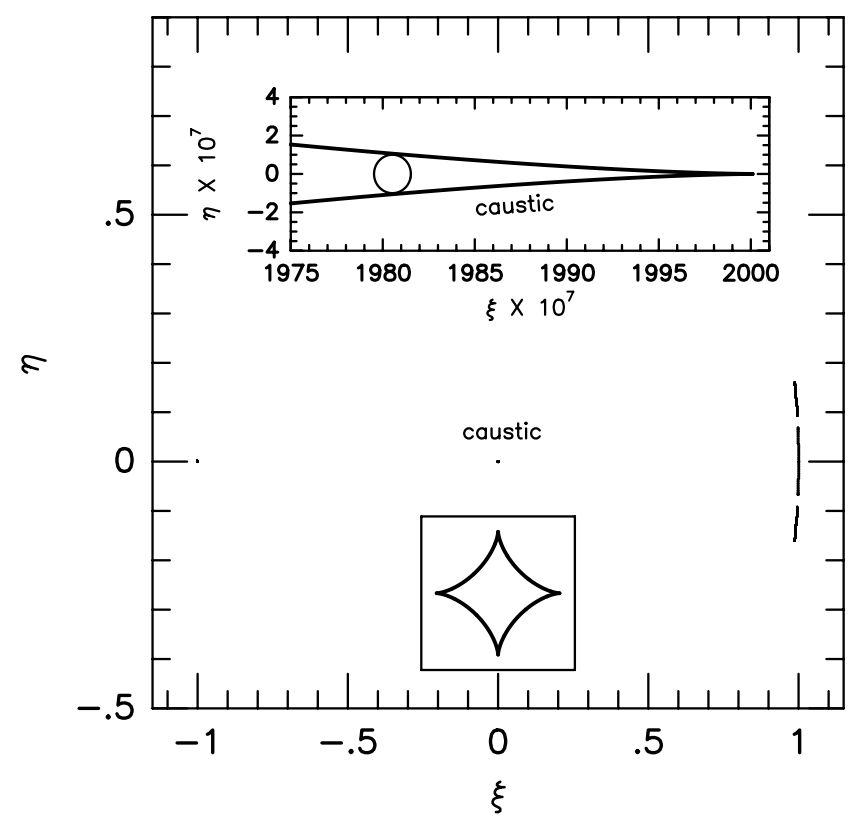

FIG. 1.-Primary lens geometry for shear $\gamma=10^{-4}$. The source lies entirely inside the caustic and near one cusp. It is therefore lensed into four images, three of which are highly magnified and lie very close to the critical curve to the right. Note that they are essentially one-dimensional. The fourth image (to the left) is not highly magnified. The lower inset shows a close up of the caustic, and the upper inset shows a highly magnified close-up of the source within the caustic.

width $10^{-6}$ mas. The relative contributions of these different regions to the observed light will scale according to their surface brightnesses. Thus, if the entire quasar had the same surface brightness, the central engine would contribute only $\sim 1 / 200$ of the light to this resolution element of the image. If surface brightness fell inversely with radius, it would contribute a fraction $1 / \ln (200) \sim 20 \%$.

There are several methods for breaking this degeneracy. The first is to make use of the three images. A most spectacular example of this approach is the recent resolution of an apparent ring galaxy using multiple images produced by

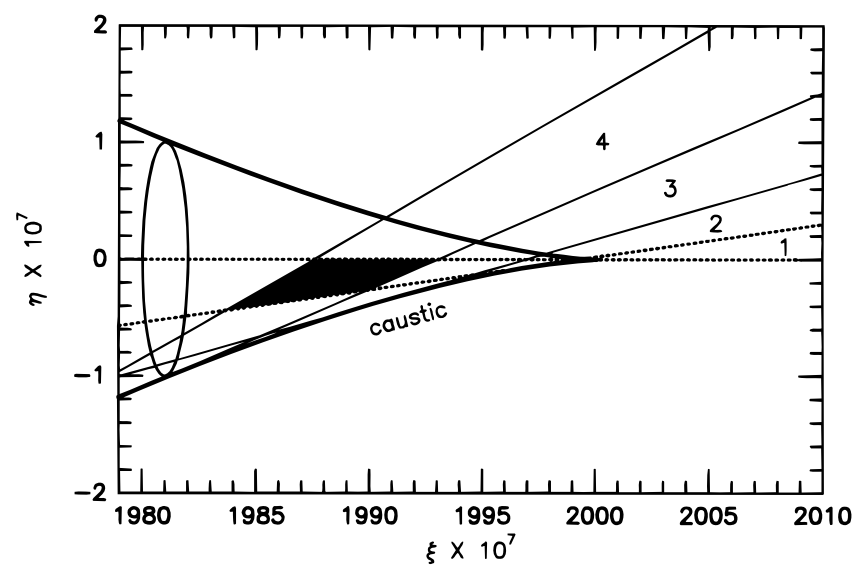

FIG. 2.-Bands of image degeneracy for shear $\gamma=10^{-4}$. The bold curve is the caustic, within which there are three images. All three images lie in an almost one-dimensional structure close to the critical curve (see Fig. 4). All the points in the source between two adjacent solid lines are mapped into the same resolution element $(\sim 0.3$ mas) along the critical-curve image. The bands are labeled 1, 2, 3, and 4. Band 1 is marked with a dotted line. Circle of radius $1 \mathrm{AU}$ representing central engine appears elliptical because the scales of the axes are not equal. Note that the central engine is resolved into $\sim 4$ resolution elements. a cluster lens (Colley, Tyson, \& Turner 1996). If there is a significant excess brightness of the middle image due to a hot central engine, this excess will also be apparent at the locations of the two other images.

There is also a second, more powerful method. As the satellite drifts over the central regions of the quasar, the point in the source that is nearest the cusp, and which is therefore maximally magnified, varies. The positions with the caustic structure of all other points in the source also vary, but the change in their magnifications with position is much smaller. Thus, one could map out the brightness of the inner regions. The second and third images would serve as a check on these measurements. Of course, this approach requires that the brightness profile of the quasar remain relatively constant during the series of observations.

\section{FEMTOLENS INTERFEROMETRY}

Both of the above methods require only that the satellite aperture be large enough $(\sim 170 \mathrm{~m})$ to resolve the images of the quasar central engine along the Einstein ring. One might think that the resolution would simply scale inversely with the aperture size. In fact, if the aperture is just a factor of a few larger, it becomes possible to resolve much smaller scales by interfering different elements of the image. Specifically, we will consider an aperture of $350 \mathrm{~m}$, which allows one to interfere resolution elements that are 0.3 mas long.

A number of authors have discussed the possibility of observing "femtolensing," interference between the integrated light of two or more images (Mandzhos 1981; Schneider \& Schmidt-Burgk 1985; Deguchi \& Watson 1986; Peterson \& Falk 1991; Gould 1992; Stanek, Paczyński, \& Goodman 1993; Ulmer \& Goodman 1995). Here we analyze the possible role of such interference in the twodimensional reconstruction of the source. The principle is relatively simple. As shown in Figure 2, each resolution element of the one-dimensional image along the critical curve corresponds to a band of variable width in the source plane. Consider the two bands labeled 1 and 4. Suppose that the light falling on the two corresponding resolution elements is brought together and then dispersed in a spectrograph. Most of the light in each resolution element comes from regions of the source that are unrelated to the regions that generate the light entering the other element. This light does not suffer any effects of interference. However, the light coming from the small shaded region where the two sets of curves cross arrives at the two images 1 and 4 at times $t_{1}$ and $t_{4}$, and therefore suffers interference according to a time lag $\Delta t_{1,4}=t_{1}-t_{4}$.

This time delay is actually a function of position within the overlapping region of the resolution elements 1 and 4 . Contours of constant delay $\Delta t_{1,4}$ are shown in Figure 3, in units of $\lambda_{0} / c$, where $\lambda_{0}=0.5 \mu \mathrm{m}$. These range from $2 \lambda_{0} / c$ to $19 \lambda_{0} / c$. Also shown are the time delays $\Delta t_{1,-4}$ between resolution elements 1 and -4 which range from $5 \lambda_{0} / c$ to $25 \lambda_{0} / c$. By taking the Fourier transform of the spectrum of the combined light of the 1 and 4 resolution elements, one could constrain the light distribution on scales of these wavenumber resolution elements. Further constraints would come from the interference of the 1 and -4 resolution elements and from the interference of the 4 and -4 elements.

The principle is illustrated in Figures 4 and 5. Figure 4 (top) shows $F(\Delta \tau)$, the fraction of all light in the overlap region contributing to interference with the time delay 


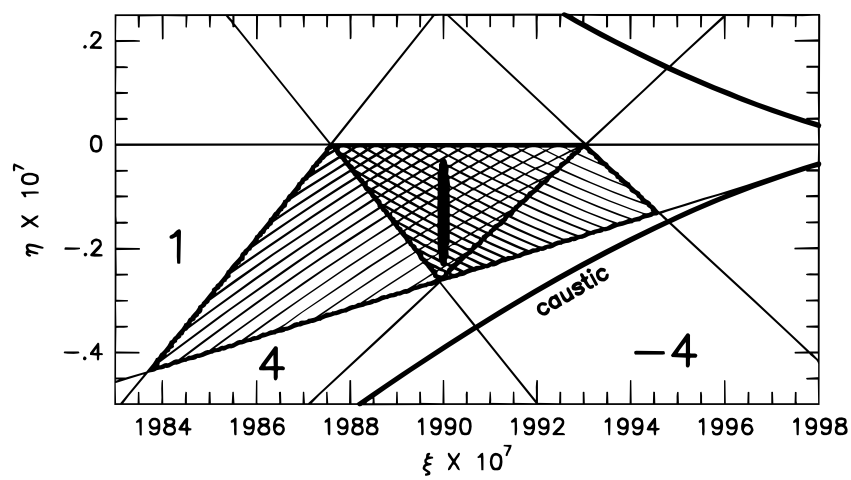

Fig. 3.-Overlap between source resolution elements 1 and 4 (see shaded region in Fig. 2) is partitioned by contours (lower left to upper right) of equal time delay between the two images $\Delta \tau_{1,4}=c \Delta t_{1,4} / \lambda_{0}$ ranging from 2 to 19 . Here $\lambda_{0}=0.5 \mu \mathrm{m}$. The overlap between source resolution elements 1 and -4 is partitioned by contours (upper left to lower right) of equal time delay $\Delta \tau_{1,-4}=c \Delta t_{1,-4} / \lambda_{0}$ ranging from 5 to 27 . A similar (approximately horizontal) set of contours could also be drawn for the delay structure between images 4 and -4 but is not shown to avoid clutter. Hypothetical circular source is shown as a filled ellipse near the center of the diagram.

$\Delta t_{1,4}=\Delta \tau \lambda_{0} / c$. Specifically,

$F(\Delta \tau)=2\left\{\frac{\int d^{2} \theta\left[A_{1}(\theta) A_{4}(\theta)\right]^{1 / 2} S(\theta) \delta\left[\Delta \tau-c \Delta t_{1,4}(\theta) / \lambda_{0}\right]}{\int d^{2} \theta\left[A_{1}(\theta)+A_{4}(\theta)\right] S(\theta)}\right\}$,

where $S(\theta)$ is the surface brightness, $A_{1}(\theta)$ and $A_{4}(\theta)$ are the magnifications of the 1 and 4 images, and $\Delta t_{1,4}(\theta)$ is the time delay between them, all as functions of angular position, $\theta$. The integration is restricted to the overlap region, and $\delta$ is the Dirac $\delta$-function. The solid curve assumes uniform surface brightness, while the dashed curve assumes that all the light comes from the circular region shown in Figure 3. The resulting spectra (normalized to the sum of the fluxes measured separately, without interference) are shown in
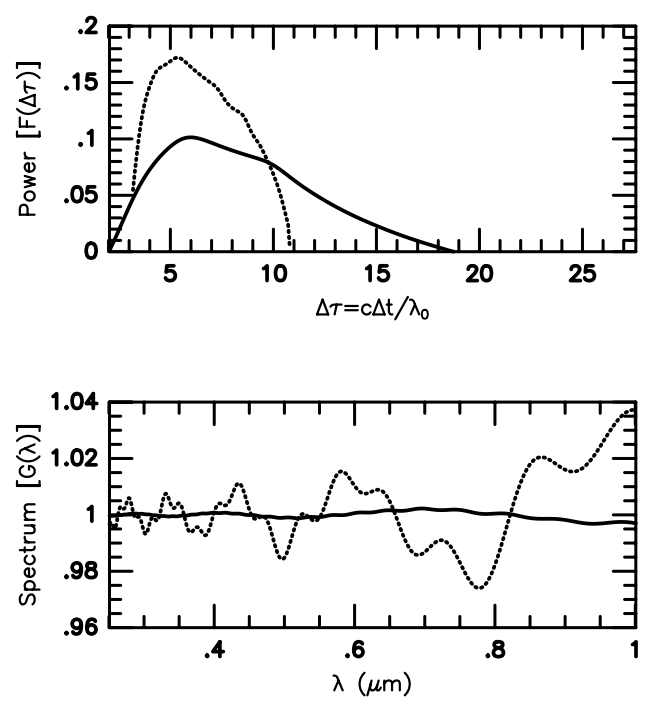

FIG. 4.-Top: Flux interfering with time delay $\Delta t_{1,4}$ between images 1 and 4 as a fraction of the total flux in the overlap region that is shown in Fig. 3. Solid curve assumes uniform surface brightness in overlap region, while dotted curve assumes that flux arises from circular source shown in Fig. 3. Time delay is normalized by $\Delta \tau_{1,4}=c \Delta t_{1,4} / \lambda_{0}$. Bottom: resulting spectra. If one observed the dotted spectrum, one could determine that the flux arose from the region of normalized time delay $3 \lesssim \Delta \tau_{1,4} \lesssim 10$.
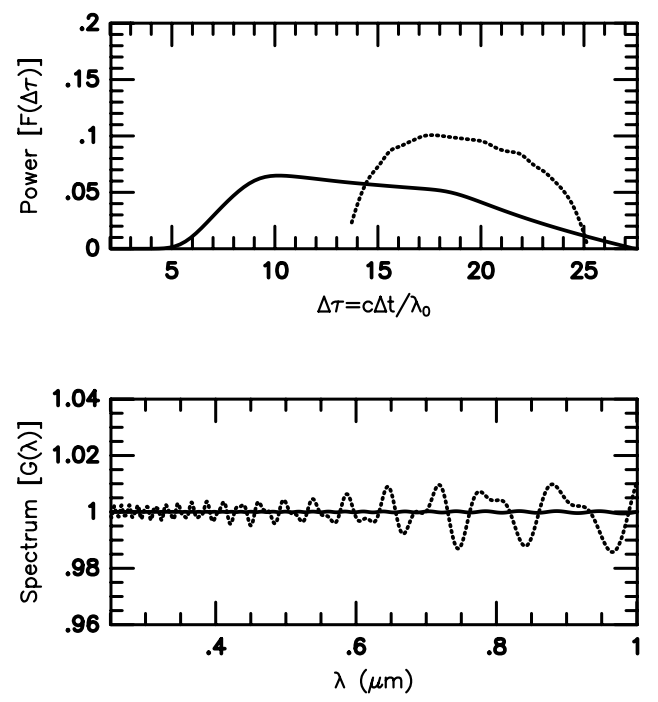

Fig. 5.-Same as Fig. 4, but for flux interfering between images 1 and -4 . If one observed the dotted spectrum, one could determine that flux arose from the region of normalized time delay $14 \lesssim \Delta \tau_{1,-4} \lesssim 25$. Combining this with the information extracted from Fig. 4, one could localize the circular source shown in Fig. 3.

Figure 4 (bottom) and are given by

$$
G(\lambda)=1+\int d(\Delta \tau) F(\Delta \tau) \cos \left(\frac{2 \pi \Delta \tau \lambda_{0}}{\lambda}\right)
$$

Note that the two spectra differ dramatically and could be distinguished, provided that the observations were sensitive to $\sim 1 \%$ variations. Note as well that high resolution is not required to observe these features, although one would want somewhat higher resolution in order to remove intrinsic spectral features of the quasar. Analogous diagrams for the interference between the 1 and -4 images are shown in Figure 5. By combining the information from the two spectra, one could determine that the flux was originating from the intervals $3 \lesssim c \Delta t_{1,4} / \lambda_{0} \lesssim 10$ and $14 \lesssim$ $c \Delta t_{1,-4} / \lambda_{0} \lesssim 25$, thereby fixing the location and size of the emitting region. Note that smaller subsources are easier to detect and are more precisely located.

\section{DISCUSSION}

It would be premature to attempt a detailed estimate of the signal-to-noise ratios in this proposed experiment. However, it is legitimate to ask whether these ratios are closer to $10^{2}$ or $10^{-2}$. Suppose that the quasar magnitude is $V=22$ and that $10 \%$ of the quasar light comes out in the annulus containing the shaded overlap region in Figure 2. The fraction of the total light produced by the overlap region is then $\sim 10^{-3}$. The typical magnifications in this region are $\sim 10^{6}$, i.e., each image has a (magnified) apparent magnitude $V \sim 14.5$. The strength of the spectral features seen in Figures 4 (bottom) and 5 (bottom) depends critically on the sharpness of the "edges" of the power spectra in Figures 4 (top) and 5 (top). The exposure time is therefore limited by the time it takes a source to move one contour interval $\left(\sim 2 \times 10^{-2} \mathrm{AU}\right)$ in Figure 3. Assuming a Keplerian speed of $\sim c / 4$ at $\sim 8$ Scharzschild radii (see Fig. 2 ) and a redshift $z \sim 2$, this is $\sim 100$ s. For definiteness, we suppose that the total area of the telescope were $20 \mathrm{~m}^{2}$. (Recall from $\S 3$ that the entire $350 \mathrm{~m}$ aperture need not be 
filled by mirrors. A one-dimensional array of $\sim 25$ mirrors, each with diameter $1 \mathrm{~m}$, would suffice.) The number of photons collected from this region during a $100 \mathrm{~s}$ exposure over a spectral range of $1 \mu \mathrm{m}$ would then be $\mathcal{O}\left(10^{8}\right)$. If there were $\sim 10$ times more photons from non-overlap regions, the signal-to-noise ratio would be $\sim 3000$, so that $\sim 1 \%$ spectral fluctuations (see Figs. 4 and 5) could be detected at the $\sim 30 \sigma$ level. To avoid "blurring" the edges of the power spectrum, it would also be necessary for the telescope to be moving slower than $100 \mathrm{~cm} \mathrm{~s}^{-1}$ relative to the caustic structure, but this condition is already met by the constraints discussed in $\S 3$. Thus it would be possible to follow the detailed evolution of the quasar on short timescales.

We have, for simplicity, focused on a specific case with shear $\gamma \sim 10^{-4}$. In estimating the binary fraction, we allowed a total range in separation of two decades, which means that the actual value of $\gamma$ might be higher or lower than our adopted value by a factor $\sim 100$. How would such a change affect one's ability to resolve the quasar? For a source of fixed size nestled in the caustic close to a cusp, the magnification scales $\propto \gamma^{-1 / 3}$. For $\gamma \sim 10^{-6}$, the quasar would be magnified $\sim 5$ times more, thus making it somewhat easier to observe. However, if the shear fell below this level, each image would take up a large fraction of the Einstein ring, in effect making it impossible to resolve multiple images. On the other hand, for $\gamma \sim 10^{-2}$, the quasar would be magnified $\sim 5$ times less, implying that a minimum aperture of $\sim 800 \mathrm{~m}$ rather than $170 \mathrm{~m}$ would be required to resolve the separate images. (In this paper, we ultimately assumed an aperture of $350 \mathrm{~m}$ in order to do femtolens interferometry.) However, if the shear were substantially larger than $10^{-2}$, the aperture size would exceed the width of the cusp, again making observations impossible. In any event, the number of binaries with separations small enough to produce such large shears is small. We conclude that the range $10^{-6}<\gamma<10^{-2}$ provides acceptable conditions for femtolens interferometry.

Since the construction of a femtolens imaging telescope lies several decades in the future, we have focused here on questions of principle rather than on practical problems. Nevertheless, we note that this project poses several significant engineering challenges. First, the energy requirements substantially exceed those of any previous satellite. While the satellite might well be able to take advantage of "slingshot" boosts from the giant planets to reach its observing position, once it got there it would still require an additional self-powered boost of $\sim 40 \mathrm{~km} \mathrm{~s}^{-1}$ to match the transverse speed of the dwarf. Second, as discussed at the end of $\S 3$, it would require additional boosts of $\sim 20 \mathrm{~cm}$ $\mathrm{s}^{-1}$ about every $10 \mathrm{hr}$. The energy needed for these is small, but stabilization of the mirror system following the boosts might prove difficult. Third, since the boosts must be repeated on timescales of the order of the light-travel time from Earth to the satellite, the satellite would need to be almost fully autonomous in its operations.
How can one find a star-quasar pair that is suitable for construction of a femtolens telescope? First, one can identify essentially all nearby (e.g., $D<30$ pc) dwarf stars from existing all-sky photographic surveys using their observed colors and apparent magnitudes and the disk mainsequence color-magnitude relation. For $\sim 75 \%$ of such stars (with $\delta>-30^{\circ}$ ), one can measure their proper motion (typically $\sim 0.3 \mathrm{yr}^{-1}$ ) from a comparison of the Palomar Observatory Sky Survey (POSS) I and POSS II plates, and hence one can determine their future positions at the time when the satellite is scheduled to reach its destination. One must then search the region within $r / D \sim 1$ ".5 of the projected positions of all these stars for quasars. That is, one would have to search a total angular area $\sim N_{Q}^{-1}=0.005 \mathrm{deg}^{2}$ to a limiting magnitude of $B=22$ to find one quasar. At this limit, there are only $\sim 20$ times more compact galaxies and stars than there are quasars, so even a single-band search would turn up only $\sim 20$ candidates. The quasars among these could be identified spectroscopically. Existing all-sky surveys reach only to $B \sim 21.5$ and so would miss $\sim 25 \%$ of the quasars, although the Sloan Digital Sky Survey (Gunn $\&$ Weinberg 1995) will go sufficiently deep over one-quarter of the sky. After a candidate pair was identified, the star could be monitored spectroscopically for duplicity. In brief, surveys would be sensitive to about half of the star-quasar pairs suitable for this experiment.

Finally, we note that a satellite telescope of this type would have at least two potential applications apart from femtolens imaging. First, it could make parallax measurements of extraordinary precision. Assuming that its diffraction-limited images could be centered to $\sim 10 \%$ of their width (i.e., $\sim 30 \mu$ as) and that the observations were coupled with others of similar quality from a telescope on or near Earth, then the parallax precision would be $\Delta \pi \sim 1$ $\mu$ as. One could, for example, determine the distance to a star in the Large Magellanic Cloud (LMC) (which happened to lie near a background quasar) to $\sim 5 \%$ accuracy from a single measurement. With multiple star-quasar pairs and multiple measurements, the distance to the LMC could be determined very precisely. Second, such a satellite could be used to measure the transverse velocities of more distant galaxies using "microlens parallaxes" (Gould 1995). For approximately three galaxies per year, a star in the galaxy will microlens a more distant quasar. The event will look significantly different as seen from the satellite at a distance $r \sim 45 \mathrm{AU}$ than it does from Earth because this distance is a substantial fraction of the Einstein radius, $r_{e} \sim 300 \mathrm{AU}$. From the difference in the light curves of the event, one can determine the transverse velocity.

We thank D. DePoy and G. Newsom for several useful suggestions. Several major issues were clarified as a result of discussions with J. Miralda-Escudé. This work was supported in part by grant AST 94-20746 from the NSF and in part by grant NAG 5-3111 from NASA.

\section{REFERENCES}

Alard, C. 1996, in IAU Symp. 173, Astrophysical Applications of Gravitational Lensing, ed. C. S. Kochanek \& J. N. Hewitt (Dordrecht: Kluwer), 215

Alcock, C., et al. 1997, ApJ, 479, 119

Chang, K., \& Refsdal, S. 1979, Nature, 282, 561

Colley, W. N., Tyson, J. A., \& Turner, E. L. 1996, ApJ, 461, L83

Deguchi, S., \& Watson, W. D. 1986, ApJ, 307, 30

Fischer, D. A., \& Marcy, J. W. 1992, ApJ, 396, 178

Gould, A. 1992, ApJ, 386, L5
Gould, A. 1995, ApJ, 444, 556

Gould, A., Bahcall, J. N., \& Flynn, C. 1996, ApJ, 465, 759

Gould, A., \& Loeb, A. 1992, ApJ, 396, 104

Gunn, J. E., \& Weinberg, D. H. 1995, in Wide Field Spectroscopy and the

Distant Universe, ed. S. Maddox \& A. Aragón-Salamanca (Singapore:

World Scientific), 3

Hartwick, R. D. A., \& Schade, D. 1990, ARA\&A, 28, 437

Labeyrie, A. 1994, A\&A, 284, 689

Mandzhos, A. V. 1981, Soviet Astron. Lett., 7, 213 
Miralda-Escudé, J. 1996, ApJ, 470, L113

Paczyński, B. 1995, Acta Astron., 45, 345

Peterson, J. B., \& Falk, T. 1991, ApJ, 374, L5

Refsdal, S. 1964, MNRAS, 128, 295

Schneider, P., Ehlers, J., \& Falco, E. E. 1992, Gravitational Lenses (Berlin: Springer)
Schneider, P., \& Schmidt-Burgk, J. 1985, A\&A, 148, 369

Stanek, K. Z., Paczyński, B., \& Goodman, J. 1993, ApJ, 413, L7

Udalski, A., et al. 1994, Acta Astron., 44, 165

Ulmer, A., \& Goodman, J. 1995, ApJ, 442, 67

Villumsen, J. V. 1996, MNRAS, 281, 369 